\title{
Métodos Computacionais e Estatísticos no Estudo das Ondas de Frequência dos Sinais Eletroencefalográficos e o Envelhecimento: uma Abordagem Através da Análise Linear do Discriminante
}

\author{
Lilian Ribeiro Mendes Paiva1 \\ Adriano Alves Pereira1 \\ Adriano de Oliveira Andrade 1
}

Resumo: O Sistema Nervoso Central (SNC) e os sinais neurológicos portam informações que representam alterações ao longo da vida. Este trabalho busca estabelecer alguma correlação entre a atividade cerebral em função da idade, a partir do registro de sinais eletroencefalográficos (EEG), em sujeitos não portadores de disfunções neurológicas, durante a prática de uma determinada tarefa. Participaram voluntariamente deste estudo 59 sujeitos saudáveis, divididos em 07 grupos, com faixa etária entre 20 a 86 anos e de ambos os sexos. Sinais EEG foram coletados em três protocolos experimentais distintos durante a execução da Espiral de Arquimedes. Os eletrodos foram posicionados conforme padrão internacional 10/20, utilizando-se os canais C3 e C4 da região central. Foram realizadas análises estatísticas para identificar diferenças de cada grupo. Os dados foram processados no software MATLAB. Dentre os resultados obtidos, foram observadas diferenças significativas, através do valor LDA. A ferramenta executou de forma satisfatória a separação de características discriminantes, classificando cada grupo de indivíduos que apresentam alta correlação em função da idade. Pode-se concluir pela análise das características utilizadas,

1Faculdade de Engenharia Elétrica, UFU.

\{Paiva, lrmpaiva@terra.com.br 
que existe a separabilidade entre os grupos conforme faixa etária, contribuindo significativamente para registrar as alterações ocorridas durante o processo de envelhecimento.

Palavras-chave: Eletroencefalografia, Análise Linear do Discriminante, Métodos estatísticos, envelhecimento.

Abstract: The Central Nervous System (CNS) and neuronal signals carry information that represents changes throughout life. This study seeks to establish some correlation between brain activity as a function of age, from the record of electroencephalographic signals (EEG), in subjects not suffering from neurological disorders, while performing a certain task. There were 59 healthy subjects that voluntarily participated in this study, which were divided into 07 groups, with ages between 20 and 86 years and both sexes. EEG signals were collected "simultaneously" in three different experimental protocols during the execution of the Spiral of Archimedes. The electrodes were positioned according to the international standard 10/20, using the channels $C 3$ and $C 4$ of the central region. Statistical analyses were performed to identify differences and allow discrimination between the characteristics of each group according to the presented changes. The data were processed with software MATLAB. Among the results, significant differences were observed, via LDA-value. The tool has satisfactorily performed the separation of discriminant features, classifying each group of individuals that have high correlation as a function of age. It can be concluded by the analysis of the characteristics used that there is the separability between groups according to age, contributing significantly to register the changes that occurred during the aging process.

Keywords: Electroencephalography, Linear Discriminant Analysis, Statistical methods, Aging. 


\section{Introdução: Abordagem Geral do Estudo}

Os sinais eletroencefalográficos (EEG) são registros gráficos da atividade elétrica do cérebro medidos pela diferença de potencial entre um eletrodo colocado sobre a superfície do córtex (sub ou sobre-cutâneo) e outro, que servirá de referência. Tais registros são a variação propagada dos potenciais pós-sinápticos de determinada região do encéfalo, somados espacialmente e temporalmente na superfície do escalpo [1], [2].

Sua representação possui variação imprevisível com forma, duração, amplitude e freqüência que portam informações, podendo ser extraídas pela análise visual qualitativa feita por especialistas ou, pela análise quantitativa, cuja leitura requer recursos computacionais e eletrônicos especialmente desenvolvidos para esse fím [2].

Um grande volume de dados é gerado pelos sistemas de monitoração de EEG, o que faz com que a análise visual completa destes dados se torne inviável na prática clínica. Com isso, surge uma grande demanda por métodos computacionais capazes de extrair, medir, analisar e classificar as características relevantes dos sinais EEG de forma automática para a realização de diagnósticos [1], [2], [3].

Luccas [3], também ressalta que a análise meramente qualitativa do sinal EEG é insuficiente para as diversas utilizações experimentais e clínicas que a neurofisiologia compreende, sendo necessárias diferentes e sucessivas transformações matemáticas para obter sua tradução num sinal mensurável e dele retirar o máximo de informações. Contudo, grandes desafios envolvem o desenvolvimento de sistemas e modelos matemáticos eficientes e compatíveis ao contexto fisiológico humano e ainda há muitos questionamentos acerca do $\mathrm{SNC}$.

Pode-se verificar a permanente evolução das técnicas eletroencefalográficas desde sua descoberta em 1929, anunciada pelo alemão Hans Berger. Nas últimas décadas, o advento do EEG digital permitiu o estudo do traçado EEG sob múltiplas perspectivas, trazendo a facilidade de armazenamento em diversos formatos e extensões, possibilitando sua análise em rede ou à distância. Atualmente, é um exame frequentemente usado como coadjuvante no diagnóstico de diversas doenças neurológicas, podendo-se verificar diferentes características desses sinais a partir dos estados funcionais do cérebro, como no sono, na anestesia, na epilepsia, nas doenças de Parkinson, Alzheimer, além de outras doenças [2].

O registro eletroencefalográfico é caracterizado por uma aparente irregularidade. Contudo, vários padrões distintos de funcionamento cerebral normal foram identificados e receberam a designação de ritmos alfa, beta, delta, gama e teta. A frequência da atividade cerebral está diretamente relacionada à atividade dos neurônios: quanto maior a energia metabólica dependida, maior será a frequência de onda registrada [4].

Quando o eletroencefalografista "lê" um EEG, evidencia-se um processo ordenado, que começa com o conhecimento de dois fatores, sem os quais a interpretação correta não é possível: a idade do paciente e o seu estado de consciência. Visualmente, o EEG de idosos sadios mantém as mesmas características gerais quando comparado ao de adultos mais 
jovens, podendo acompanhar-se de pequenas alterações, como aumento da atividade beta $\mathrm{e}$ diminuição da reatividade alfa [5], [6], [7], [8], [9].

Canineu [5], Damasceno [10] e Ottaviano [11] relatam diferenças de individuo para individuo conforme hereditariedade, meio ambiente, dieta, estilo de vida e o hábito de praticar exercícios físicos, dentre outras particularidades. Mota [12] complementa que a senilidade ocorre de formas adversas segundo idade biológica, social e psicológica, que podem ser muito diferentes da idade cronológica, sendo o EEG importante na avaliação do envelhecimento cerebral para o reconhecimento de alterações cerebrais.

Nesta abordagem entre EEG e envelhecimento, este estudo investiga as diferenças significativas entre os ritmos de frequências de sinais EEG coletados durante a realização de uma tarefa específica: o desenho da Espiral de Arquimedes, para identificar a separabilidade entre grupos de indivíduos normais, segundo o decorrer da idade, através de métodos estatísticos e computacionais.

\section{Materiais e Métodos}

Participaram voluntariamente, 59 sujeitos saudáveis, sem evidências clínicas de problemas neurológicos, conforme avaliação realizada em consulta com médico neurologista. Todos os integrantes também responderam a um questionário para identificação de seus hábitos de vida, além de assinarem um termo de consentimento aprovado pelo Comitê de Ética em Pesquisa da Universidade Federal de Uberlândia, Brasil, registrado sob o número 188/06, do Parecer do Comitê de Ética em Pesquisa, número 354/06. Os voluntários foram distribuídos em 07 grupos, tendo como critério cada década, conforme tabela 01.

Tabela 1: Distribuição dos voluntários que participaram das coletas, segundo grupo, idade e gênero.

\begin{tabular}{ccccc}
\hline Grupo & Faixa Etária & Homens & Mulheres & Total \\
\hline 01 & $20-29$ & 07 & 03 & 10 \\
02 & $30-39$ & 08 & 02 & 10 \\
03 & $40-49$ & 06 & 03 & 09 \\
04 & $50-59$ & 02 & 06 & 08 \\
05 & $60-69$ & 03 & 07 & 10 \\
06 & $70-79$ & 02 & 06 & 08 \\
07 & $80-89$ & 02 & 02 & 04 \\
\hline
\end{tabular}


Observa-se na tabela 1 e figura 1, que o número de sujeitos no grupo 07 é relativamente pequeno quando comparado aos demais grupos de análise. Esta diferença é justificada por dificuldades no recrutamento de indivíduos nessa faixa etária (80-89 anos), conforme os critérios definidos para o estudo: indivíduos saudáveis, sem problemas neurológicos e que não fizessem uso de medicamentos durante o período da pesquisa.

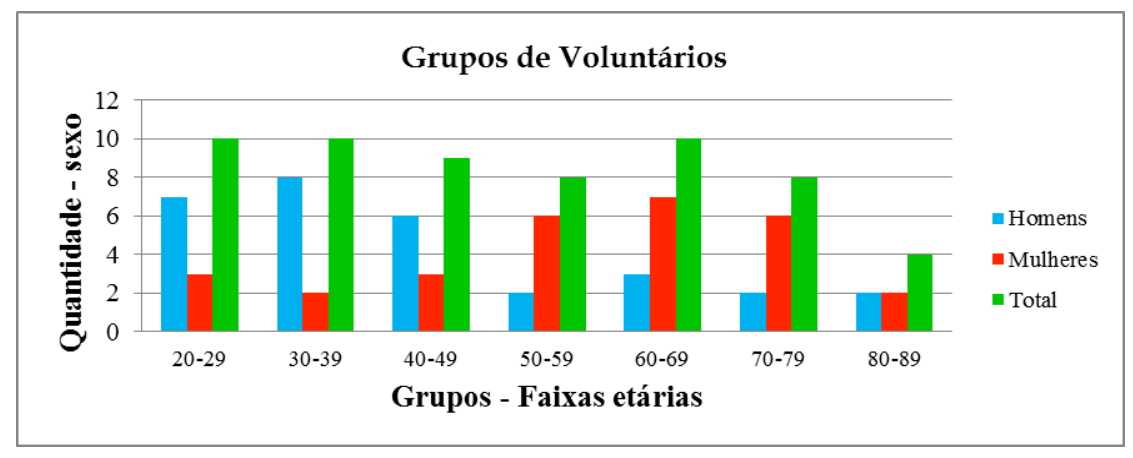

Figura 01: Distribuição de voluntários por gênero e faixas etárias

\subsection{Sistema de Eletroencefalografia}

O equipamento utilizado para coleta do sinal EEG foi o BrainNet BNT-36, um amplificador e condicionador de sinais biológicos de 36 canais, adequado à aquisição de sinais eletroencefalográficos e poligráficos para aplicações em monitoramento e eletro diagnóstico. Sua função foi amplificar e converter os sinais elétricos gerados pelo cérebro em sinal digital e enviá-lo para a entrada de dados do computador no qual foram armazenados e analisados. Outras partes que compõe o sistema de eletroencefalografia são: fonte de alimentação, cabo de conexão entre o BrainNet-36 e o computador, programa BrainTech de aquisição e análise, eletrodos e/ou sensores e massa condutora.

A figura 2 ilustra resumidamente as etapas de processamento dos sinais e como as características utilizadas nesse trabalho foram extraídas dos sinais coletados com os equipamentos, analisadas e quantificadas por meio de ferramentas estatísticas e algoritmos desenvolvidos na ferramenta matemática computacional MATLABC.

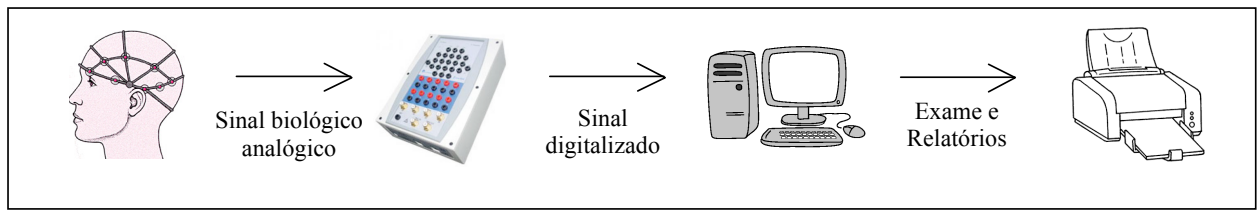

Figura 02: Exemplo de aplicação do BrainNet-36 em um sistema de eletroencefalografia 


\subsection{Protocolo de Coleta dos Sinais EEG}

O protocolo de coleta dos experimentos realizados nesta pesquisa foi definido cuidadosamente, o que possibilitou a uniformização dos procedimentos e a aquisição de sinais de qualidade. Os sujeitos foram instruídos a desenhar duas amostras da Espiral de Arquimedes com sua mão predileta, conforme ilustrado na figura 3, que apresenta uma visão geral dos procedimentos. A primeira amostra foi coletada com o sujeito desenhando a espiral a partir do seu centro até a extremidade (outgoing spiral - OS), enquanto a segunda amostra foi desenhada pelo sujeito a partir da extremidade da espiral até o seu centro (ingoing spiral - IS) e, posteriormente, permanecendo parado no centro da espiral. Este procedimento foi repetido três vezes para cada sujeito. Recomendou-se aos voluntários que desenhassem as espirais com uma velocidade que lhes fosse natural.

Dentre os desenhos mais utilizados para a análise de sinais, a espiral de Arquimedes ganha destaque na avaliação neurológica de pacientes e, sua utilização, constitui uma técnica conhecida como espirografia. Esta técnica consiste na reprodução, pelo paciente, da espiral de Arquimedes de acordo com um modelo ideal. Observa-se sua ampla utilização para diversos fins como citado em Almeida [13].

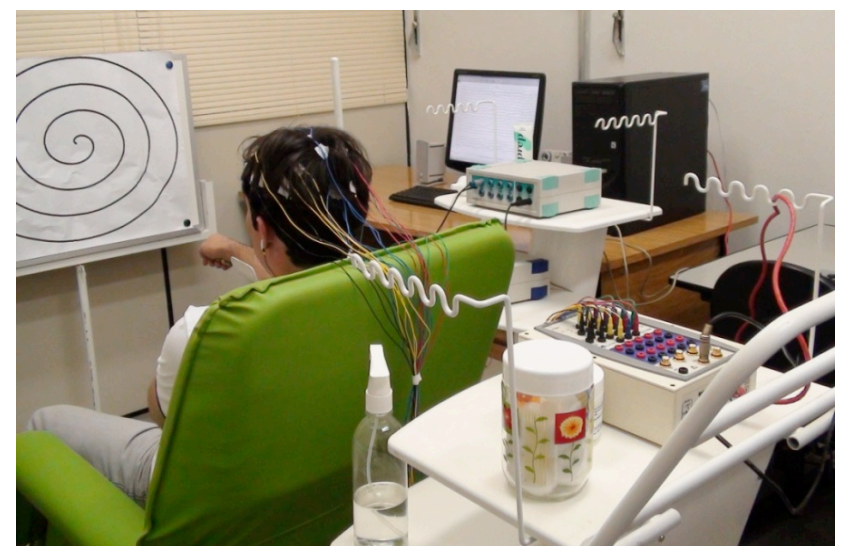

Figura 3: Voluntário da pesquisa, realizando o exame

Os procedimentos e protocolos de coleta estão demonstrados na figura 6 e no detalhamento de cada etapa:

a) Nesta etapa os eletrodos foram posicionados no escalpo do voluntário. Adotou-se a eletroencefalografia superficial, método não invasivo e indolor de coleta dos sinais EEG, com posicionamento conforme o sistema 10/20, recomendado pela Federação Internacional da Sociedade de Encefalografia e Neurofisiologia. Antes de iniciar o experimento foi realizada a calibração dos canais, através do software que 
acompanha o equipamento (Braintech for Windows V3.0) para garantir precisão, estabilidade e imunidade de interferências nos sinais.

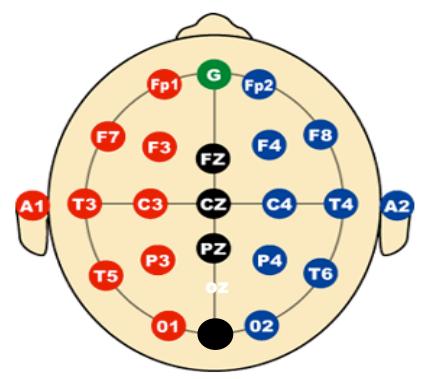

Figura 4: Demonstração do posicionamento dos eletrodos de acordo com o sistema 10/20 recomendado pela Federação Internacional das Sociedades de Encefalografia e Neurofisiologia.

b) Posicionamento dos sujeitos em uma cadeira, em frente a um quadro branco com desenho da Espiral de Arquimedes em uma posição confortável para realização do procedimento e regulagem da altura do pedestal, de forma a permitir que o ângulo entre o tronco e o braço preferido do paciente fosse de 90 graus. As figuras 3 e 5 ilustram essas especificações, assim como a visão geral do experimento.

c) Posicionamento do laser pointer no centro da espiral, informações ao voluntário quanto ao exato momento de iniciar o desenho da espiral e ativação do equipamento de sincronização.

d) Repouso de 20 segundos. Durante este lapso temporal, devidamente contabilizado, o paciente mantinha uma posição estática, de forma a manter o sinal emitido pelo laser posicionado no centro do desenho modelo.

Todo o processo foi repetido três vezes. O tempo de cada coleta foi de aproximadamente 30 segundos e o tempo médio para realização deste primeiro exame foi de 60 minutos para cada sujeito incluindo posicionamento de eletrodos e realização das tarefas. É importante ressaltar, que dois desenhos distintos de espirais estavam disponíveis. Um destinado ao exame com a mão direita e outro para a mão esquerda.

\subsection{Pré-processamento dos Sinais EEG}

Através dos sinais aquisicionados e na representação de componentes específicos, foram realizados processamentos matemáticos, para o estudo do sinal EEG no domínio do tempo e no domínio da frequência, que permitissem esclarecer informações relevantes e associar resultados numéricos com os dados, para subsequente comparação. 


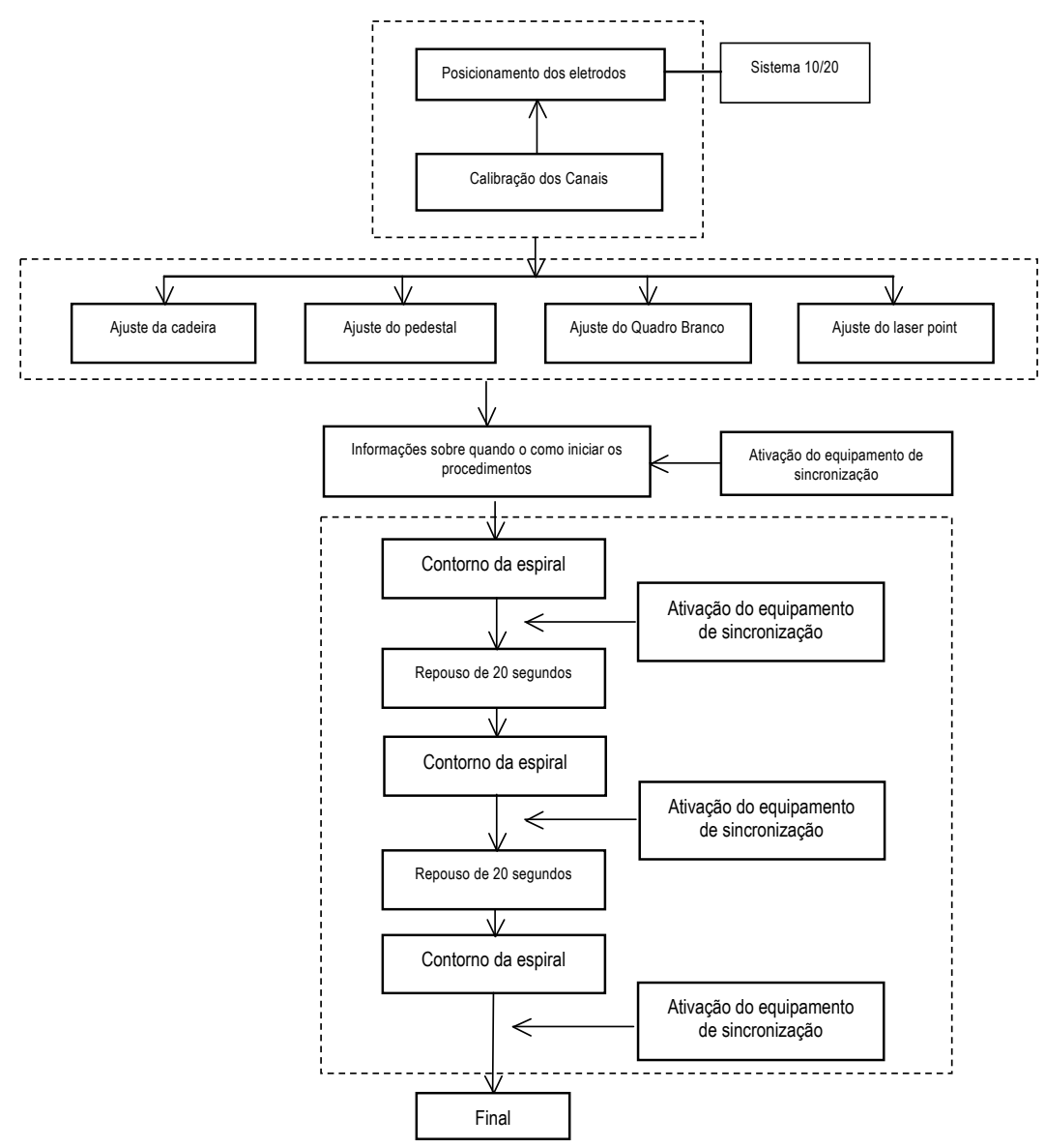

Figura 5: Diagrama referente às etapas dos procedimentos e protocolos de coleta.

Segundo Parreira [14], a predominância de um determinado tipo de onda, depende da região do escalpo que está sendo analisada e do estado em que o indivíduo se encontra. Conforme padronização estabelecida por Barbosa [15], Nidermeyer[16], Stern[17],Tatum[18] e Teplan[2] o sinal EEG foi subdividido em diferentes faixas de ondas descritas na tabela 2, onde são demonstrados exemplos de situações em que tais ritmos predominam caracteristicamente:

Tabela 2: Ondas cerebrais e situações em que surgem como ritmo dominante 
Estudo de Sinais Eletroencefalográficos e o Envelhecimento: Análise Linear do Discriminante

\begin{tabular}{ccc}
\hline Ritmo & $\begin{array}{c}\text { Componente de } \\
\text { frequência }\end{array}$ & Condição do individuo \\
\hline Delta $(\delta)$ & $(0.5 \mathrm{a} 3.5 \mathrm{~Hz})$ & Estado de sono profundo \\
Theta $(\theta)$ & $(3.6 \mathrm{a} 7.9 \mathrm{~Hz})$ & Sonolência e sono superficial \\
Alfha $(\alpha)$ & $(8-13 \mathrm{~Hz})$ & Vigília com relaxamento \\
Beta $(\beta)$ & $(13-30 \mathrm{~Hz})$ & Vigília com estado de atenção \\
Gama $(\gamma)$ & $(>30 \mathrm{~Hz})$ & Vigília com estado de programação \\
& & motora \\
\hline
\end{tabular}

Fonte: [15], [16],[17] e [18].

(a)

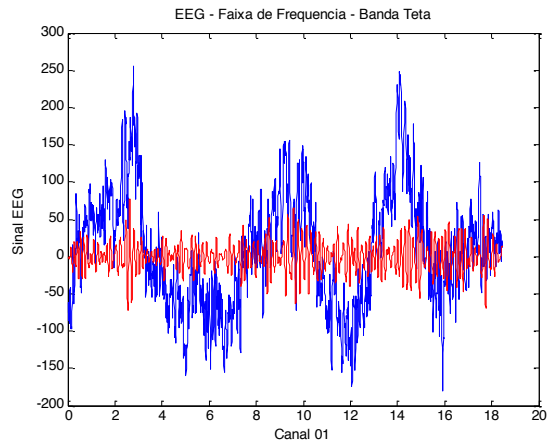

(b)

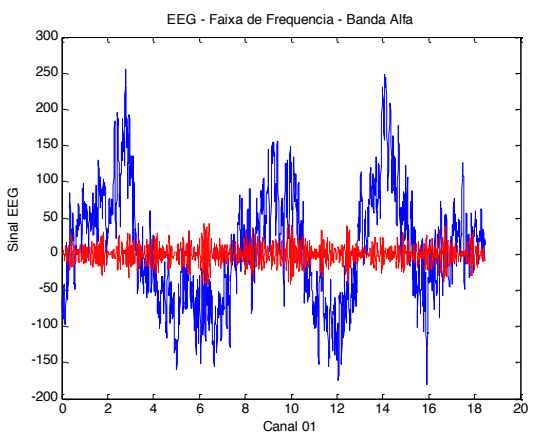

Figura 06: Sinal EEG, (a) banda Theta (04 a $08 \mathrm{~Hz})$ e (b) banda Alfa (08 a $13 \mathrm{~Hz})$.

(a)

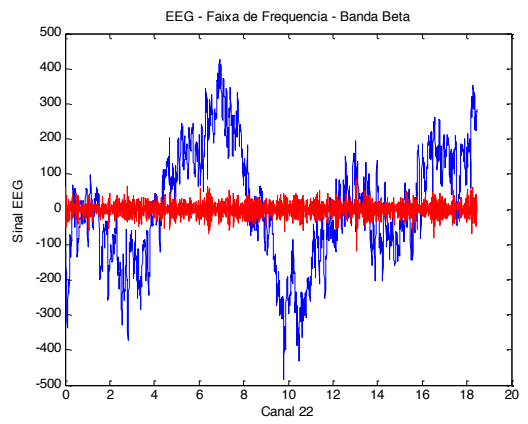

(b)

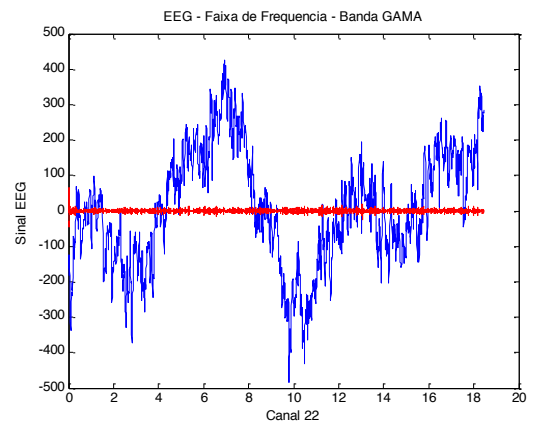

Figura 07: Sinal EEG (a) banda Beta (13 a $40 \mathrm{~Hz}$ ), (b) banda Gama (40 a $100 \mathrm{~Hz}$ ) 
As figuras 6 e 7 mostram o sinal completo (traço azul) e as faixas de frequência teta, alfa, beta e gama (traço vermelho) filtradas a partir do sinal completo. Podem ser visualizadas alterações de amplitude em função de cada faixa de frequência como representação dos estados de concentração, relaxamento, atenção e estado de programação motora.

Para análise quantitativa dos sinais EEG, foram analisados os sinais dos canais C3 e $\mathrm{C} 4$, escolhidos segundo área que supostamente apresentaria aumento de função nos casos de realização de uma tarefa específica e suas etapas, conforme dita o referencial teórico sobre a aquisição, o processamento e o controle de uma habilidade motora [19], [20], [21], [22].

Através de análises estatísticas foram comparadas as características extraídas dos sinais EEG, registrados digitalmente entre os 07 grupos. Estas comparações foram feitas entre variáveis isoladas e conjuntamente em: bandas de frequência $(50 \%, 80 \%, 90 \%$ e 95\%), frequência média, raiz média quadrática (RMS), cruzamento em zero, energia do sinal, curtose, assimetria, variância, desvio padrão e entropia aproximada [23], [24], [25], [26], [27], [28].

Posteriormente, conforme recomendado pela literatura, a análise da variância (ANOVA) foi aplicada em cada característica de cada protocolo para determinar se a diferença observada entre as médias era significativa, mediante as variações obtidas [29], [30], [31]. Assim, mesmo com médias diferentes, pode-se para verificar se as diferenças são suficientes para serem consideradas relevantes. Uma probabilidade ( $p$-value) menor que 0.05 $(\mathrm{p}<0.05)$ foi definida como limiar de significância.

Porém, apenas através da correlação entre as características tradicionais com a idade dos sujeitos não foi possível verificar a correlação significativa com a idade. Observou-se que os dados obtidos não foram úteis para representar a separabilidade dentre as classes de dados, ficando os mesmos representados de forma não linear, espalhados ao longo de uma única superclasse. Para resolver este problema, utilizou-se o LDA, como um critério estatístico que maximiza a separação entre classes e minimiza o espalhamento entre as mesmas.

\subsection{Análise Linear do Discriminante (LDA)}

É um método para classificação e redução dimensional de dados, supondo que grupos ou classes são linearmente separáveis e, sendo possível estimar novas características, projetadas em eixos otimizados, que maximizem a separabilidade entre as classes [32], [33], [34]. Pode ser utilizado para melhorar o desempenho das técnicas estatísticas, além de verificar a relevância das características tradicionais.

O valor LDA foi estimado para estabelecer uma possível correlação linear entre as características com a idade dos sujeitos. A técnica utilizada nesta pesquisa para estimativa do valor LDA foi também descrita por Cavalheiro et al. [35], em um estudo que analisa a 
relação entre controle postural e envelhecimento, empregando Algoritmos Genéticos (AG), como método de busca para solucionar os problemas de otimização.

O algoritmo encontra-se ilustrado na figura 8, partindo inicialmente de uma matriz de dados, sendo necessário normalizá-la para que não haja influência das diferentes escalas das características nas análises. Cada fator, representado pelas colunas da matriz, foi normalizado entre 0 e 1, assim como cada resultado, para evitar a divisão por zero nas etapas posteriores que consistiram:

- Representação dos dados, matriz normalizada, em um espaço com coordenadas angulares multidimensionais.

- Projeção dos dados em um eixo particular, resultando em um único escalar, ou em uma nova característica.

- Início da aplicação do AG com a definição de uma população inicial, criada a partir da amostragem de eixos imaginários. Através do AG foi possível encontrar os ângulos de rotação, que caracterizam a máxima separabilidade entre as classes.

- $\quad$ projeção do conjunto de dados multidimensionais sobre um espaço unidimensional um conjunto de projeções que é utilizado para cálculo do estimador de acurácia.

- Calculo da função de aptidão do AG, para cada eixo imaginário. O objetivo do AG é encontrar os ângulos de rotação para os quais esse valor será máximo.

- Seleção pela técnica da roleta, um método de amostragem com reposição comumente usada em AG, para selecionar aleatoriamente indivíduos de uma geração para criação da base da próxima geração [36, 37, 38].

- Geração de três descendentes, onde apenas os dois melhores são selecionados conforme valores de suas funções de aptidão.

- Mudança aleatória de alguns indivíduos resultando em uma nova população.

- Localização do eixo imaginário que maximiza a separação de classes e a relevância das características utilizadas na análise.

- Repetição de todo o processo quando o eixo é encontrado, sendo a relevância de cada fator verificada calculando-se um novo valor, mas com o fator ajustado para zero. 


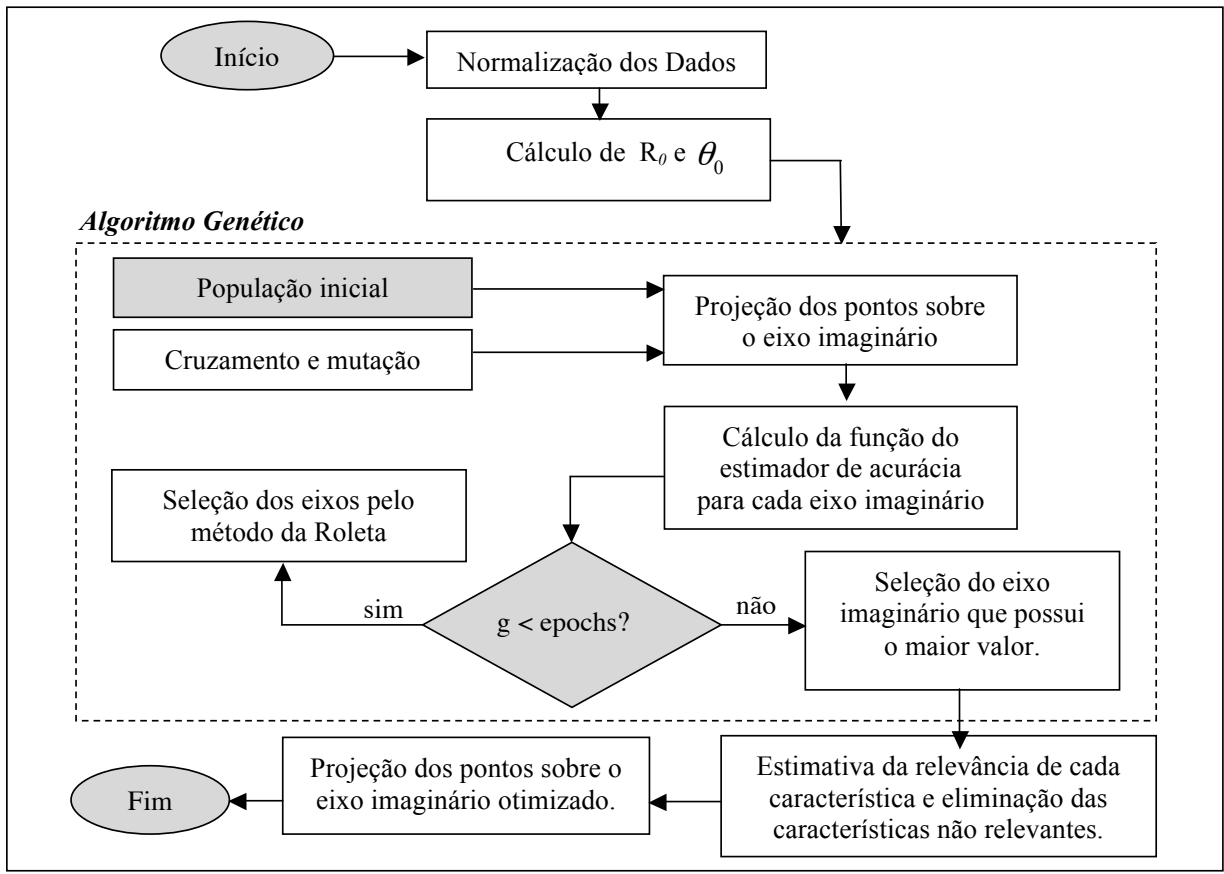

Figura 8: Diagrama ilustrando os principais passos para cálculo do LDA.

\section{Resultados e Discussão}

O valor LDA foi estimado para buscar uma possível correlação linear entre as características com a idade dos sujeitos.

Para discussão e análise dos resultados, utilizou-se o Boxplot que é um gráfico estatístico que possibilita estudar o comportamento de variáveis, sendo importante para analisar a simetria de uma distribuição, o espalhamento das observações e a presença de observações discrepantes. 
As figuras 9,10,11, 12 e 13 mostram os valores LDA obtidos para os sete grupos em cada faixa de frequência. Observam-se resultados similares para faixas etárias iguais, quando as mesmas representam protocolos diferentes.
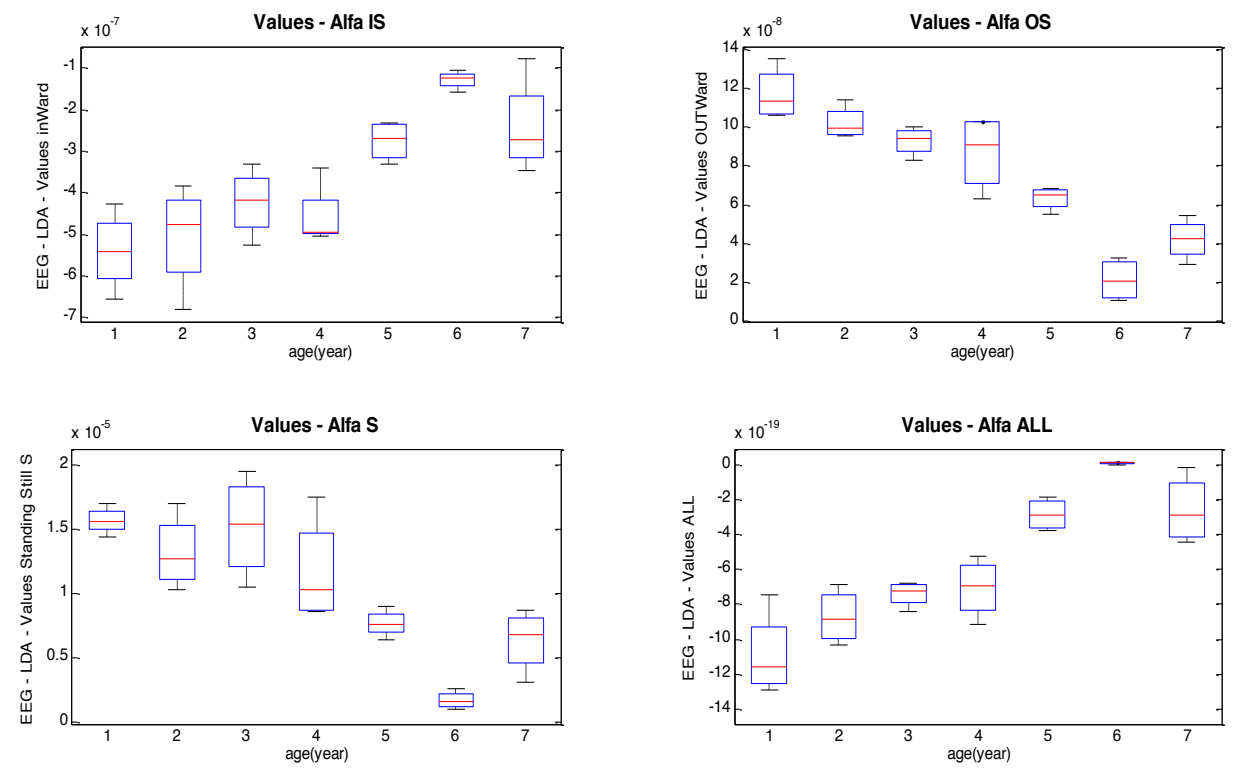

Figura 9: Gráfico box plot dos LDA-values obtidos para os sete grupos durante cada protocolo (IS, OS e repouso) e todos os protocolos analisados em conjunto, na frequência Alfa.

A análise visual do gráfico box plot permite concluir que o valor LDA é uma característica que tem seu valor modificado com a idade. As linhas sólidas das caixas são as medianas do valor LDA calculadas para os 07 grupos estudados. Estes resultados mostram a separabilidade entre os mesmos e alterações ocorridas conforme faixa etária. Observa-se a não existência dos símbolos (+) que representando os outliers, ou observações atípicas, destoantes dos demais valores. 

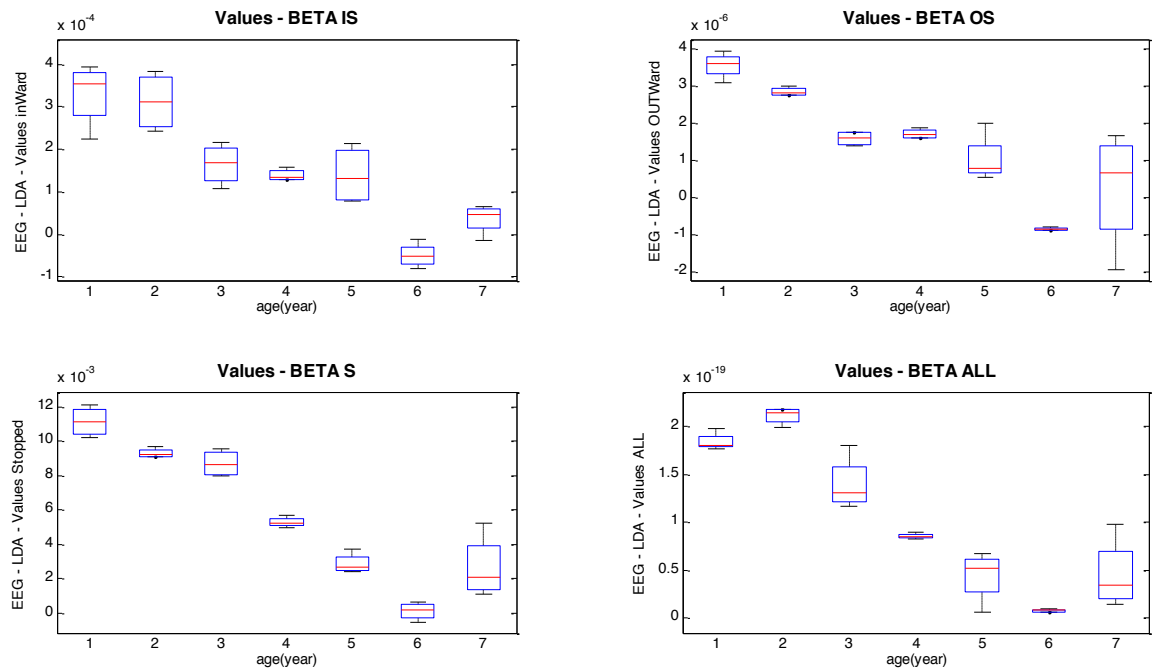

Figura 10: Gráfico box plot dos LDA-values obtidos para os sete grupos durante cada protocolo (IS, OS e repouso) e todos os protocolos analisados em conjunto, na frequência Beta.
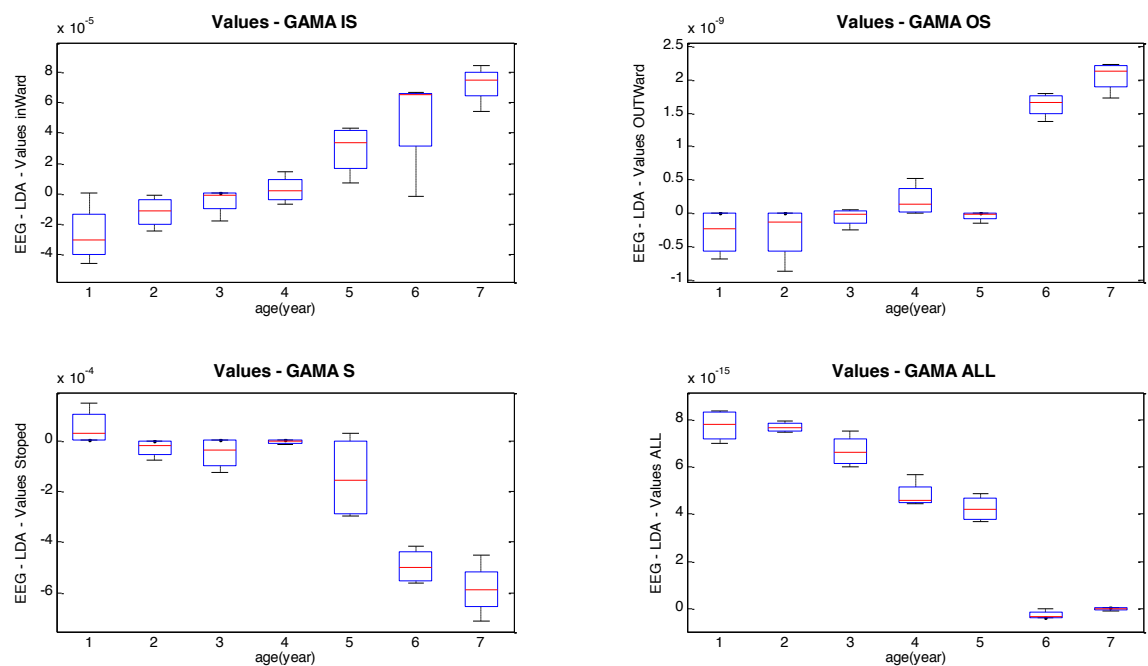

Figura 11: Gráfico box plot dos LDA-values obtidos para os sete grupos durante cada protocolo (IS, OS e repouso) e todos os protocolos analisados em conjunto, na frequência Gama. 

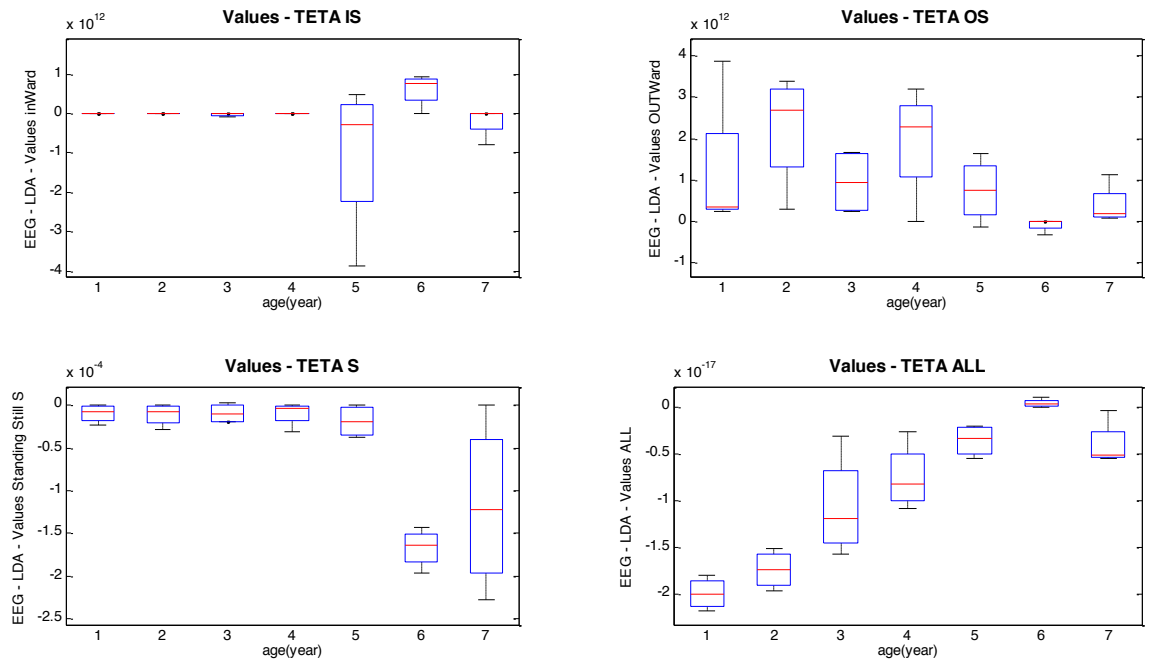

Figura 12: Gráfico box plot dos LDA-values obtidos para os sete grupos durante cada protocolo (IS, OS e repouso) e todos os protocolos analisados em conjunto, na frequência Teta.
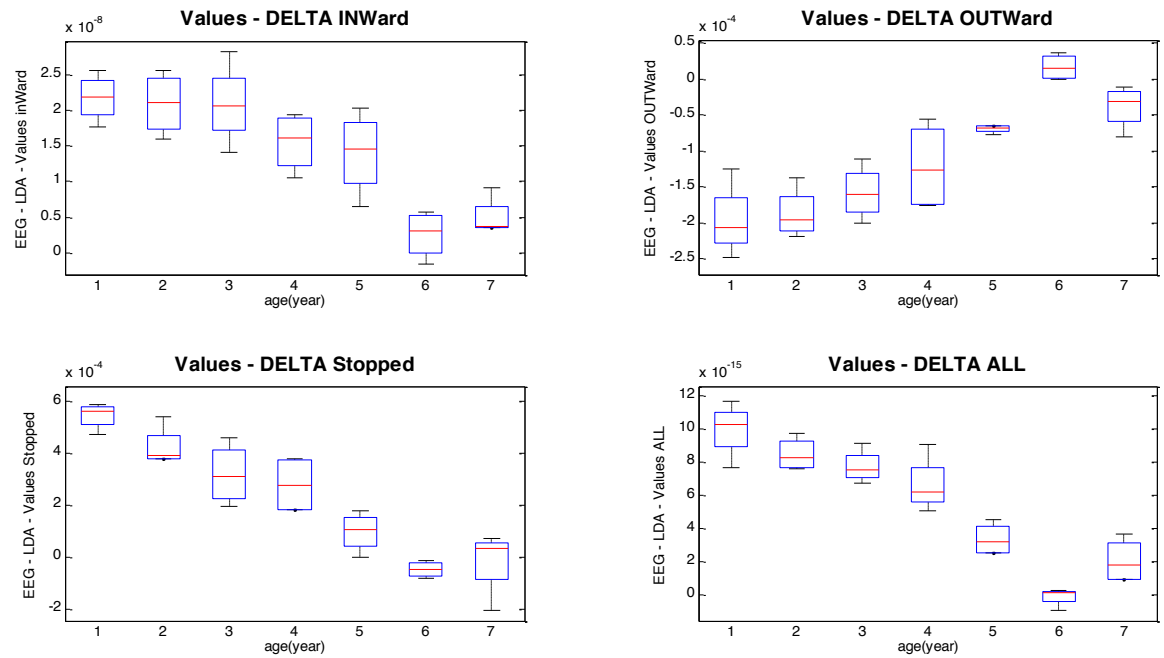

Figura 13: Gráfico box plot dos LDA-values obtidos para os sete grupos durante cada protocolo (IS, OS e repouso) e todos os protocolos analisados em conjunto, na frequência Delta. 
Analisando as caixas do gráfico da Figura 13, observa-se que as linhas medianas, vão decrescendo em função da idade apenas no processamento conjunto dos 03 movimentos. Destaca-se nos movimentos Ingoing Spiral (IS), sentido de desenho da extremidade para o centro, Outgoing Spiral (OS), sentido de desenho do centro para a extremidade e parado (P), maior distanciamento entre as linhas medianas representando valores menores obtidos para o coeficiente de correlação de Pearson.

A faixa de frequência de cada sinal EEG possui uma representatividade, sendo que em adultos saudáveis sinalizam diferentes estados como vigilância e sono, além de características que também mudam com idade. O coeficiente de correlação de Pearson apresentou valores descritos na tabela 3 .

Tabela 3: Valores do coeficiente de correlação de Pearson em cada ritmo de frequência e protocolo.

\begin{tabular}{clr}
\hline Componente de Frequência & \multicolumn{1}{c}{ Protocolos } & Valor LDA \\
\hline & Ingoing Spiral (IS) & 0.79 \\
Delta & Outgoing Spiral (OS) & 0.85 \\
$(0.5$ a 3.5 Hz) & Parado (P) & 0.92 \\
& Todos os Protocolos (TP) & 0.90 \\
& Ingoing Spiral (IS) & 0.76 \\
Theta & Outgoing Spiral (OS) & 0.80 \\
$(3.6$ a $7.9 \mathrm{~Hz})$ & Parado (P) & 0.72 \\
& Todos os Protocolos (TP) & 0.89 \\
& Ingoing Spiral (IS) & 0.85 \\
Alfha & Outgoing Spiral (OS) & 0.85 \\
$(8-13 \mathrm{~Hz})$ & Parado (P) & 0.83 \\
& Todos os Protocolos (TP) & 0.90 \\
& Ingoing Spiral (IS) & 0.84 \\
Beta & Outgoing Spiral (OS) & 0.77 \\
$(13-30 \mathrm{~Hz})$ & Parado (P) & 0.81 \\
& Todos os Protocolos (TP) & 0.89 \\
& Ingoing Spiral (IS) & 0.82 \\
Gama & Outgoing Spiral (OS) & 0.81 \\
$(>30 \mathrm{~Hz})$ & Parado (P) & 0.83 \\
& Todos os Protocolos (TP) & 0.90 \\
\hline
\end{tabular}




\section{Conclusão}

As elaborações de estudos clínicos ou experimentais são a base do desenvolvimento de qualquer área do conhecimento. Dessa forma, a evolução da ciência está diretamente ligada à produção de informações de qualidade e amplamente disponíveis e, neste aspecto, os recursos da informática especialmente no que tange à captura, armazenamento e busca de dados clínicos, tem sido de suma importância para geração de estudos clínicos relevantes e confiáveis[ 40], [41].

Neste trabalho, foi demonstrada a análise quantitativa dos sinais EEG e o estudo desta correlação com a idade, para separabilidade de grupos de voluntários, entre diferentes faixas etárias (entre 20 e 80 anos).

Para tanto, o EEG captou ondas no tempo real de exposição à tarefa proposta (desenho da Espiral de Arquimedes, em três protocolos distintos: a partir do seu centro até a extremidade (outgoing spiral - OS), a partir da extremidade da espiral até o seu centro (ingoing spiral - IS), parado no centro da espiral) em relação às funções das regiões onde estavam colocados os eletrodos no escalpo, canais C3 e C4, de grupos de sujeitos agrupados em classes conforme suas idades.

Os resultados indicaram que o LDA foi eficaz na quantificação dos sinais, mostrando um alto grau de correlação para os diferentes protocolos. Como o valor LDA é linearmente correlacionado com a idade, este índice pode ter grande importância em pesquisas futuras, particularmente naquelas relacionadas com a discriminação entre sinais EEG fisiológicos e patológicos.

Observa-se no conjunto dos trabalhos investigados nesta pesquisa a intensa utilização de análises por meio do LDA, demonstrando ser um método estatístico consolidado [35], [39]. Porém, as abordagens aqui descritas, investigaram diferentes formas de se interpretar e utilizar as informações, não somente sob o ponto de vista da redução da dimensionalidade, como também de correlação entre as características em função da idade (envelhecimento).

Foram verificadas diferenças significativas entre os grupos de jovens e idosos mostrando a correlação e alterações no sinal EEG em função da idade dos indivíduos. Essa conclusão mostra tanto a validade do LDA como ferramenta de análise, quanto, a necessidade de se utilizarem outros métodos estatísticos para ampliar as possibilidades de observação dos resultados obtidos nesta pesquisa.

Ehlers e Kupfer [38] concordam que o EEG de idosos sadios mantém as mesmas características gerais de adultos mais jovens. Assim, apesar de indicar redução na frequência e amplitude, propensão ao elentecimento do ritmo alfa, fragmentação e descontinuidade correspondente a um índice de deterioração mental, a experiência clínica diária apresentada nos estudos de Teplan[2], Barbosa[15] e Nitish[30], mostram que os indivíduos principalmente após os 80 anos de idade, mas em boa saúde, apresentam traçados impossíveis de serem diferenciados qualitativamente dos de um indivíduo jovem. Neste 
contexto, esta pesquisa inicialmente apresenta de forma quantitativa, a possibilidade de diferenciar, ou separar estes traçados através da metodologia utilizada.

As conclusões apontadas pelos resultados obtidos com a utilização do LDA demonstram que é possível aprofundar ainda mais nas pesquisas sobre as alterações provocadas pela idade no organismo humano, auxiliando especialistas na busca por novas ferramentas e soluções que promovam a melhoria na qualidade de vida da população, principalmente em idosos.

\section{Referencias}

[1] OISHI, N. et al. Neural correlates of regional EEG power change. NeuroImage, vol. 36, pp. 1301-1312, 2007.

[2] TEPLAN, M. Fundamentals of EEG Measurement. Measurement Science Review, vol. 1, pag. 11, 2001.

[3] LUCCAS, F. J. C., et al. Recomendações para Registro e Interpretação do Mapeamento Topográfico do Eletroencefalograma e Potenciais Evocados, Arq Neuropsiquiatr, vol. 57, pp. 132-146, 1999.

[4] STERN, J. M. ; ENGEL, J. Atlas of EEG patterns. Lippincott Williams \& Wilkins, 307 p., 2004.

[5] CANINEU, P. R. Ageing and the Changes to the Nervous System, Biblioteca Virtual em Saúde vol. 21, 1997.

[6] ALHO, C. S. Genética Molecular e Envelhecimento. In: FREITAS, E. V. et al (Ed.). Tratado de Geriatria e Gerontologia. Rio de Janeiro: Guanabara Koogan, 2006, p.2334.

[7] KIRKWOOD, T. B.; AUSTAD, S. N. Why do we age? Nature, n. 408, p. 233-238, 9 nov. 2000.

[8] NERI, A. L. Desenvolvimento e Envelhecimento: Perspectivas Biológicas, Psicológicas e Sociológicas. Papirus, 200p. 2007.

[9] TROEN, B. R. The Biology of Aging. The Mount Sinai Journal of Medicine v. 70, n. 1, p. 3-22, 2003.

[10] DAMASCENO, B. P. Envelhecimento Cerebral - O problema dos limites entre o normal e o patólogico, Arq Neuropsiquiatr, vol. 57(1), pp. 78-83, 1999

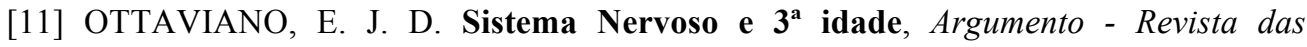
Faculdades de Educação Ciências e Letras e Psicologia Padre Andchieta, vol. 4, pag.:116, 2000. 
[12] MOTA, M. P. et al., Biological Theories of Aging, Revista Portuguesa de Ciências do Desporto, vol. 4, pp. 81-110, 2004.

[13] AlMEIDA, M.F.S.; CAVALHEIRO, G.L.; FURTADO, D.A.; PEREIRA, A.A.; ANDRADE, A.O. Investigation of changes in kinetic tremor through analysis of hand-drawing movements - Differences between physiological and essential tremors. In: IV International Joint Conference on Biomedical Engineering Systems and Technologies, 2011, Roma, Itália. Proceedings of the International Conference on Bioinspired Systems and Signal Processing, pp.393-398, 2011. DOI 10.5220/0003121703930398.

[14] PARREIRA, F. J. Detecção de Crises Epiléticas a Partir de Sinais Eletroencefalográficos. Doutorado, Faculdade de Engenharia Elétrica, Universidade de Uberlândia - UFU, Uberlândia, 2006.

[15] BARBOSA, A. F. \& Medeiros A. A. D. : Relatório de Estágio Supervisionado: Interface Cérebro Computador off-line Baseada em Eletroencefalograma, Centro de Tecnologia. Departamento de Engenharia de Computação e Automação. Universidade Federal do Rio Grande do Norte, Natal, p. 54, 2009.

[16] NIEDERMEYER, E.; Silva, F. L.. Electroencephalography: basic principles, clinical applications, and related fields - Lippincott Williams \& Wilkins, ISBN: 0-7817-51268, Pag.:139-159, 2005.

[17] STERN, J. M. ; ENGEL, J. Atlas of EEG patterns. Lippincott Williams \& Wilkins, 307 p., 2004.

[18] TATUM, W. O. Handbook of EEG Interpretation. Demos Medical Publishing, 276 p., 2007.

[19] BLAnCO, S. G. R.; Quiroga, L. Q.; Romanelli, L. Stationarity of the EEG Series. IEEE Engineering in Medicine and Biology, 1995.

[20] CARSKadon, M. A. e Rechtschaffen. A. Monitoring and Staging Human Sleep. In: Kryger, M., Roth, T. e Dement, W. Principles and Practice of Sleep Medicine. 2. Ed. USA: W. B. SaundersCompany, p 943 -955, 1994.

[21] ROCHA, Ana Clara B. et al., Observação das evidências cognitivas de aprendizagem motora no desempenho de jovens violonistas monitoradas por eletroencefalograma: um estudo piloto. Ciências \& Cognição. Vol 14 (1), pp. 103 $120,2009$.

[22] BENEVIDES, A. B. Classificação de Padrões Mentais Via Redes Neurais Artificiais e Transformada WAVELET. Faculdade de Engenharia Eletrica, CentroTecnológico da Universidade Federal do Espírito Santo, Vitoria, 2007.

[23] BRUHN, J., et al. : Approximate Entropy as an Electroencephalographic Measure of Anesthetic Drug Effect during Desflurane Anesthesia, American Society of Anesthesiologists, Inc., vol. 92(3), pp. 715-726, 2000 
[24] CARVALHO, S. : Estatística Geral e Aplicada. Rio de Janeiro, 2004.

[25] DEVASAHAYAM, S. R. : Signals and Systems in Biomedical Engineering vol. 01. New York, 2000.

[26] GAÁL, Zsófia Anna et al., Age-dependent features of EEG-reactivity - Spectral, complexity, and network characteristics, Neuroscience Letters, vol. 479, pp. 79-84, 2010 .

[27] HAYKIN, S., VAN VEEN, B. Sinais e Sistemas. Tradução de José Carlos Barbosa dos Santos. Porto Alegre: Bookman, 668p.2001.

[28] MASSIMILIANO, Ignaccolo et al. : The dynamics of EEG entropy, Journal of biological physics vol. 36(2), pp. 185-1962010.

[29] SANEI, S.; CHAMBERS, J. EEG signal processing. Wiley-Interscience, 289 p., 2007.

[30] NITISH V. T. \& TONG, S. Advances in Quantitative Electroencephalogram Analysis Methods, Annual. Rev. Biomedical. Eng., vol. 6, pp. 453-95, 2004.

[31] MONTGOMERY, D. C. : Applied Statistics and Probability for Engineers. United States of América: Wiley, 2006.

[32] PINCUS, SM: Approximate entropy as a measure of system complexity. Proceedings of the National Academy of Science 1991, 1991:2297-2301.

[33] IGNACCOLO, M. et al. : Dynamics of EEG Entropy: beyond signal plus noise, Physics arXiv:0902.1113, 2009.

[34] NUNES, R. R.: Entropy: A New Method of Measuring Depth of Anesthesia.Comparative Study with Bispectral Index during Clinical Evaluation in Tracheal Intubation of Patients Anesthetized with Sevoflurane, Revista Brasileira de Anestesiologia, vol. 54(3), pp. 289 - 302, 2004.

[35] CAVAlHeIRO, G., M. F. S. Almeida, A. Pereira and Andrade, A. O.: Study of agerelated changes in postural control during quiet standing through Linear Discriminant Analysis. BioMedical Engineering OnLine 8, 2009.

[36] AKAY, M. : Detection and Estimation Methods for Biomedical Signals vol. 01. San Diego, 1996.

[37] AKAY, M. : Biomedical Signal Processing vol. 01. San Diego, 1994.

[38] EHLERS C.L., KUPFER D. J. Effects of age on delta and REM sleep parameters. Electroencephalogr Clin Neurophysiol. 72:118-125, 1989.

[39] ALMEIDA, M., G. Cavalheiro, A. Pereira and Andrade, A. O. : Investigation of AgeRelated Changes in Physiological Kinetic Tremor. Annals of Biomedical Engineering 38:3423-3439, 2010. 
[40] FARINATTI, P. T. V.: Teorias biológicas do envelhecimento:do genético ao estocástico, Revista Brasileira Med Esporte, vol. 8, p. 10, 2002.

[41] YOUNG, A. Ageing and physiological functions, Phil.T rans. R. Soc. Lond. B pp. 1837-1843, 1997. 